Short communication

\title{
Identification and expression analysis of duck interleukin-17D in Riemerella anatipestifer infection
}

\author{
Joyce Anne R. Diaz ${ }^{\mathrm{a}, 1}$, Woo H. Kim ${ }^{\mathrm{a}, 1}$, Cherry P. Fernandez ${ }^{\mathrm{a}}$, Jipseol Jeong ${ }^{\mathrm{b}}$, \\ Fahmida Afrin a, Hyun S. Lillehoj ${ }^{\text {c }}$, Suk Kim ${ }^{\text {a }}$, Sungwon Kim ${ }^{\mathrm{d}}$, Rami A. Dalloul ${ }^{\mathrm{d}}$, \\ Wongi Min a,* \\ a College of Veterinary Medicine \& Institute of Animal Medicine, Gyeongsang National University, Jinju, 52828, Republic of Korea \\ ${ }^{\mathrm{b}}$ Environmental Health Research Division, National Institute of Environmental Research, Incheon, 22689, Republic of Korea \\ c Animal Biosciences and Biotechnology Laboratory, Agricultural Research Service, United States Department of Agriculture, Beltsville, MD, 20705, USA \\ ${ }^{\mathrm{d}}$ Avian Immunobiology Laboratory, Department of Animal and Poultry Sciences, Virginia Tech, Blacksburg, VA, 24061, USA
}

\section{A R T I C L E I N F O}

\section{Article history:}

Received 25 February 2016

Received in revised form

4 April 2016

Accepted 4 April 2016

Available online 6 April 2016

\section{Keywords:}

Ducks

Interleukin-17D

Riemerella infection

Avian IL-17D

\begin{abstract}
A B S T R A C T
Interleukin (IL)-17D is a proinflammatory cytokine with currently largely unknown biological functions. Here we provide the description of the sequence, bioactivity, and mRNA expression profile of duck IL-17D homologue. A full-length duck IL-17D (duIL-17D) cDNA with a 624-bp coding region was identified from the large intestine. duIL-17D shares approximately $94.7 \%$ identity with its chicken counterpart, which is also identified in this work. duIL-17D exhibits 62.6-68.4\% and 52.1-53.1\% identity with mammalian and piscine homologues. Recombinant duIL-17D promoted the expression of proinflammatory cytokines such as IL-6, IL-8, and IL-1 $\beta$ in duck embryo fibroblast cells. Very low levels of duIL-17D transcript were observed in healthy lymphoid tissues, including bursa, thymus, and spleen, while duIL-17D expression was relatively high in the heart. The duIL-17D expression profiles were examined in mitogen-stimulated splenic lymphocytes, as well as tissues affected by Riemerella anatipestifer infection. The levels of duIL17D were mostly upregulated in mitogen-activated splenic lymphocytes but downregulated in the liver and spleen of $R$. anatipestifer-infected ducks. These results provide new insights into the roles of IL17D in host protective immune responses to Riemerella infection, which can therefore lead to further studies of its biological functions in different disease models of ducks and other avian species.
\end{abstract}

(c) 2016 Elsevier Ltd. All rights reserved.

\section{Introduction}

The interleukin (IL)-17 cytokine family plays a broad role in the host immune response and the pathogenesis of multiple inflammatory disorders (Iwakura et al., 2011; Pappu et al., 2012). Six IL-17related cytokines (IL-17A, IL-17B, IL-17C, IL-17D, IL-17E, and IL-17F) have been identified in mammals, each with a varying degree of intermolecular amino acid sequence homology and functional activities. These cytokines generally function through engagement of the IL-17 receptor (IL-17R) family, which includes five members (IL17RA, IL-17RB, IL-17RC, IL-17RD, and IL-17RE), by binding as homodimers or heterodimers (Onishi and Gaffen, 2010; Mensikova

\footnotetext{
* Corresponding author. College of Veterinary Medicine, Gyeongsang National University, 501 Jinju-daero, Jinju, Gyeongnam, 52828, Republic of Korea. E-mail address: wongimin@gnu.ac.kr (W. Min).

1 Joyce Anne R. Diaz and Woo H. Kim contributed equally to this work.
}

et al., 2013). IL-17A and IL-17F are the most intensively studied members of the family and are produced by activated $\mathrm{T}$ cells and a variety of innate cells to mediate protective immunity against microbial pathogens, as well as the pathogenesis of autoimmune diseases (Iwakura et al., 2011; Gu et al., 2013; Min et al., 2013). Recently, IL-17C was shown to have similar activities as IL-17A and IL-17F even though IL-17C is expressed mainly by epithelial cells and was found to be a critical factor in mucosal immunity (RamirezCarozzi et al., 2011; Song and Qian, 2013). IL-17E enhances type 2 immune responses by promoting the production of Th2 cytokines, such as IL-4, IL-5, and IL-13, and contributes to host defense against parasitic infections (Gu et al., 2013; Song and Qian, 2013). Although IL-17B and IL-17D play a similar role in inducing inflammatory mediators such as IL-17A and IL-17F, their biological functions remain largely unknown.

IL-17D shares the highest sequence homology with IL-17B and is known to be preferentially expressed outside the immune system. 
IL-17D reportedly stimulates the expression of IL-6, IL-8, and granulocyte-macrophage colony-stimulating factor (GM-CSF) in human vascular endothelial cells, and exerts an inhibitory effect on the hematopoiesis of myeloid progenitor cells (Starnes et al., 2002). In contrast, IL-17D expression is decreased in psoriatic skin (Johansen et al., 2009) and undetectable in human synovial fluid mononuclear cells (Hwang and Kim, 2005). Additionally, a significant increase in IL-17D mRNA was detected in certain unedited tumors, but not in edited mouse tumor cell lines (O'Sullivan et al., 2014). In infection experiments, IL-17D mRNA expression was significantly enhanced in the head kidney but not in the spleen of Atlantic salmon that were injected intraperitoneally with Aeromonas salmoncida (Kumari et al., 2009). This effect was also observed in the head kidney of grass carp intraperitoneally infected with Aeromonas hydrophila (Du et al., 2014).

The avian immune system provides an important model for the study of basic and applied immunology. Despite the general lack of cross-reactivity and the low level of sequence conservation between avian and mammalian cytokines (Gibson et al., 2012; Jeong et al., 2015; Stewart et al., 2013), our understanding of avian immunobiology increased extensively over the past decade. Recently, the growing literature on the critical roles of the IL-17/IL$17 \mathrm{R}$ axis in host immune responses includes many reports that describe the importance of the IL-17 family of cytokines in avian immunity (Hong et al., 2008; Kim et al., 2012, 2014; Min et al., 2013; Rychlik et al., 2014; Zhao et al., 2014). Given the role that IL-17D plays in various diseases, understanding its potential contribution in avian health is of considerable interest. In this study, we report the discovery and characterization of a full-length cDNA encoding a duck homologue of mammalian IL-17D. By also examining the expression and bioactivity of this cytokine, we sought to gain better insight into its function in ducks. Finally, using quantitative realtime RT-PCR (qRT-PCR), we evaluated the expression profile of duck IL-17D (duIL-17D) in birds infected with Riemerella anatipestifer, one of the major pathogens afflicting the duck industry.

\section{Materials and methods}

\subsection{Animals and infections}

Pekin ducklings (Joowon ASTA Ducks, South Korea) were given unlimited access to feed and water. Constant light was provided throughout the experiment. Fifty two-week-old ducks were infected intramuscularly with $5 \times 10^{7} \mathrm{CFU}$ of $R$. anatipestifer serotype 7 in $200 \mu \mathrm{l}$ phosphate buffer saline (PBS). The bacterium used in this study (Jeong et al., 2014) was isolated from a commercial duck farm in Changwon, Gyeongnam, South Korea and serotyped in Chonbuk National University. The isolate was grown in sheep blood agar plates (Asan Pharmaceutical, Korea) at $37{ }^{\circ} \mathrm{C}$ in $5 \% \mathrm{CO}_{2}$. A single isolated colony was picked and inoculated into Tryptic Soy Broth (Difco, USA) and incubated at $37^{\circ} \mathrm{C}$ in a shaking incubator until the logarithmic growth phase was achieved. The final inoculum concentration was determined by plating 10 -fold serial dilutions onto sheep blood agar plates. Control birds were given the same volume of PBS as the volume of bacteria used for infection. Liver and spleen were collected from five birds per group at $1,4,7$, and 10 days postinfection. All animal experiments were performed in accordance with the Gyeongsang National University Guidelines for the Care and Use of Experimental animals and approved by the Institutional Animal Care and Use Committee (IACUC) of Gyeongsang National University, Jinju, Republic of Korea (GNU-150504-C0026).

\subsection{Cloning of duIL-17D and sequence analysis}

Total RNA was extracted from the large intestine of a healthy duck using RiboEx (GeneAll, Korea) and RNeasy Mini Kit (Qiagen, Germany). The RNA was then treated with RNase-free DNAse I (Fermentas, Canada) and cDNA was synthesized from the treated total RNA using the first strand synthesis kit, HyperScript (GeneAll). Based on a predicted cDNA sequence (GenBank accession number XM_005028731.2), 5'/3'-Rapid Amplification of cDNA Ends (RACE) was performed using duIL-17D-specific primers (Table 1) with the large intestine cDNA, a high-fidelity DNA polymerase (Bioneer, Korea), and a 5'/3' RACE kit (5'/3' RACE kit, 2nd Generation; Roche Applied Science, Germany) according to the manufacturer's instructions. PCR products were cloned into a TA vector (RBC, Taiwan) and sequenced (Macrogen, Korea). PCR was performed on a DNA Engine thermocycler (Bio-Rad, USA) with the following program: $5 \mathrm{~min}$ at $95{ }^{\circ} \mathrm{C} ; 35$ cycles of $1 \mathrm{~min}$ at $95{ }^{\circ} \mathrm{C}, 1 \mathrm{~min}$ at $55^{\circ} \mathrm{C}$, and $1 \mathrm{~min}$ at $72{ }^{\circ} \mathrm{C}$; and a final 5 min extension at $72{ }^{\circ} \mathrm{C}$. duIL-17D cDNA sequence was submitted to GenBank (accession number KT932633). Protein identification was conducted using the Expert Protein Analysis System (ExPASy; www.expasy.org/tools/) and multiple amino acid alignments were generated using Clustal Omega (http://www.ebi.ac.uk/Tools/msa/clustalo/). A signal peptide was predicted using the Signal P 4.1 Server (http://www.cbs. dtu.dk/services/SignalP/) and the potential $N$-linked glycosylation site was determined by the NetNGlyc 1.0 Server (http://www.cbs. dtu.dk/services/NetNGlyc/).

\subsection{Cell culture}

Duck splenic lymphocytes isolated from two-week-old ducks and duck embryonic fibroblast cells (DEF) obtained from ten-dayold embryos were cultured as previously described (Jeong et al., 2015; Kim et al., 2015). Cells were collected, washed twice with PBS, and cultured in DMEM (Gibco Life Technologies, USA) supplemented with $10 \%$ fetal bovine serum (Gibco Life Technologies) and penicillin-streptomycin $(10,000 \mathrm{unit} / \mathrm{ml})$ in a $41^{\circ} \mathrm{C}$ incubator in $5 \% \mathrm{CO}_{2}$. Splenic lymphocytes were resuspended to $5 \times 10^{6}$ cells $/ \mathrm{ml}$ and stimulated with $25 \mu \mathrm{g} / \mathrm{ml}$ polyinosinic:polycytidylic acid (poly $\mathrm{I}: \mathrm{C}), 10 \mu \mathrm{g} / \mathrm{ml}$ lipopolysaccharide (LPS from Escherichia coli, O111:B4) (all purchased from Sigma-Aldrich, Germany), or $10 \mu \mathrm{g} / \mathrm{ml}$ concanavalin (ConA) (Amersham Bioscience, Sweden) for 0, 4, 8, and $24 \mathrm{~h}$. DEF was stimulated with the conditioned medium from COS-7 cells transfected with duIL-17D-FLAG or empty vector (pcDNA 3.1) for $6 \mathrm{~h}$ at $41{ }^{\circ} \mathrm{C}$ in $5 \% \mathrm{CO}_{2}$, and then proinflammatory cytokine expression was analyzed by qRT-PCR.

\subsection{Production and biological activity of recombinant duIL-17D}

duIL-17D cDNA harboring a FLAG-tag expression sequence (duIL-17D-FLAG) was amplified by PCR from single-stranded cDNA isolated from duck large intestine using the following specific primers: forward 5'-GATCGAATTCATGCAGCGAGGCAGGGTGCT- ${ }^{\prime}$ and reverse $5^{\prime}$-GATCCTCGAGTCACTTGTCGTCATCGTCTTTGTAGTCTTCTGAAGATGGCTTGTT-3'. The primers contained EcoRI and XhoI restriction enzyme sites (underlined with solid lines above) and a FLAG-expressing sequence (underlined with a dashed line). PCR products were digested with EcoRI and XhoI and cloned into the corresponding restriction sites of the pcDNA $3.1+$ vector (Invitrogen, USA). COS-7 cells were transiently transfected with $10 \mu \mathrm{g}$ of the construct or empty vector (negative control) using FuGENE 6 transfection reagent (Promega, USA) according to the manufacturer's instructions. Transfected cells were cultured in Opti media (Gibco Life Technologies, USA), a serum-free medium, for $48 \mathrm{~h}$ at $37{ }^{\circ} \mathrm{C}$ in $5 \% \mathrm{CO}_{2}$. To assess the biological activity of recombinant duIL-17D, primary DEF was obtained from ten-day-old embryos and cultured as described above. Supernatants from transfected COS-7 cells were added to DEF and then incubated for 
Table 1

List of primers used in cloning and quantitative real-time RT-PCR.

\begin{tabular}{|c|c|c|c|}
\hline Gene target & Purpose & Primer and sequence $\left(5^{\prime}-3^{\prime}\right)$ & References \\
\hline duIL-17D & 5'RACE & (Fwd) GATGACGGTGGGCATGTACA & Present study \\
\hline & 3'RACE & (Rev) CCCGAAGCGTACTGCCTGTG & \\
\hline duIL-17D & qRT-PCR & $\begin{array}{l}\text { (Fwd) CACGAGATACCCTAAGTACATTC } \\
\text { (Rev) CTGCGGAAGTGGAAATTCT }\end{array}$ & Present study \\
\hline duIL-17A & qRT-PCR & $\begin{array}{l}\text { (Fwd) ATGTCTCCAACCCTTCGT } \\
\text { (Rev) CCGTATCACCTTCCCGTA }\end{array}$ & Kim et al. (2015) \\
\hline IL-6 & qRT-PCR & $\begin{array}{l}\text { (Fwd) GCGGTCTCCGACTCCTCC } \\
\text { (Rev) ATAGCGAACAGCCCTCACG }\end{array}$ & Wei et al. (2014b) \\
\hline IL-8 & qRT-PCR & $\begin{array}{l}\text { (Fwd) GCTGTCCTGGCTCTTCTCCT } \\
\text { (Rev) GCACACCTCTCTGTTGTCCTTC }\end{array}$ & Wei et al. (2014b) \\
\hline IL-1 $\beta$ & qRT-PCR & $\begin{array}{l}\text { (Fwd) TCATCTTCTACCGCCTGGAC } \\
\text { (Rev) GTAGGTGGCGATGTTGACCT }\end{array}$ & Wei et al. (2014a) \\
\hline TGF- $\beta$ & qRT-PCR & $\begin{array}{l}\text { (Fwd) CGGGACGGATGAGAAGAAC } \\
\text { (Rev) CGGCCCACGTAGTAAATGAT }\end{array}$ & Shanmugasundaram and Selvaraj (2012) \\
\hline$\beta$-actin & qRT-PCR & $\begin{array}{l}\text { (Fwd) GCTATGTCGCCCTGGATTTC } \\
\text { (Rev) CACAGGACTCCATACCCAAGAA }\end{array}$ & Liu et al. (2012) \\
\hline
\end{tabular}

(Fwd): Forward primer; (Rev): Reverse primer; qRT-PCR: quantitative real-time RT-PCR.

$6 \mathrm{~h}$ at $41^{\circ} \mathrm{C}$ in $5 \% \mathrm{CO}_{2}$. Total RNA was extracted from the stimulated cells and cDNA synthesis was performed using oligo (dT) primer and the first strand synthesis kit, HyperScript (GeneAll). Analysis of duIL-17D-induced gene expression was performed by qRT-PCR using the primers listed in Table 1.

\subsection{Western blot analysis}

Cell lysates and supernatants of transfected COS-7 cells were mixed with equal volumes of sample buffer $(0.125 \mathrm{M}$ Tris- $\mathrm{HCl}, \mathrm{pH}$ 6.8, 4\% SDS, 20\% glycerol, 10\% 2-mercaptoethanol, and $0.004 \%$ bromophenol blue), heated for $5 \mathrm{~min}$ at $95^{\circ} \mathrm{C}$, resolved on $12 \%$ SDSpolyacrylamide gels, and electroblotted onto polyvinyl difluoride (PVDF) membrane (Bio-Rad, USA). The membrane was blocked with a PBS and $0.05 \%$ Tween-20 (PBS-T) solution containing $4 \%$ nonfat dry milk for $2 \mathrm{~h}$ at room temperature and then incubated with monoclonal anti-FLAG mouse antibody (Cell Signaling Technology, USA) at $4{ }^{\circ} \mathrm{C}$ overnight. The membrane was then washed with PBS-T five times and incubated with horseradish peroxidaseconjugated goat anti-mouse IgG antibody (Promega) in 4\% nonfat dry milk in PBS-T for $1 \mathrm{~h}$ at room temperature. The membrane was then washed five times with PBS-T. It was then incubated with chemiluminescent reagent EZ West Lumi plus (Atto, Japan) and visualized using the ChemiDoc Imaging System (Bio-Rad).

\subsection{Cytokine induction assay and quantitative real-time RT-PCR (qRT-PCR)}

Healthy tissues, activated splenic lymphocytes, and tissue samples from ducks infected with $R$. anatipestifer serotype 7 were subjected to qRT-PCR analysis in duplicate. RiboEx (GeneAll) was used to extract total RNA from samples homogenized with a tissue grinder (Daihan Sci. Korea) or by vortexing cells according to manufacturer's instructions. It was then purified with the RNeasy Mini kit (Qiagen) and stored at $-70{ }^{\circ} \mathrm{C}$. To remove any contaminating genomic DNA, samples were treated with DNase I (Fermentas). Single-stranded cDNA was synthesized with the first strand synthesis kit, HyperScript (GeneAll), from the total RNA using oligo (dT) primer. The qRT-PCR was performed using the CFX 96 real-time PCR system (Bio-Rad, USA) with SYBR Green (Bioneer, South Korea) and the primers listed in Table 1. A melting curve was obtained at the end of each run to verify the presence of a single amplification product without primer dimers. Relative expression levels of individual transcripts were normalized to those of $\beta$-actin using Bio-Rad CFX software as previously described (Kim et al.,
2015).

\subsection{Statistical analysis}

Data were analyzed by the Student's $t$-test using InStat ${ }^{\circledR}$ software (GraphPad, USA). Differences with a $p$-value less than 0.05 were considered statistically significant. Data are expressed as the mean \pm standard error $(\mathrm{SE})$.

\section{Results}

\subsection{Cloning and characterization of duIL-17D}

The full-length cDNA encoding a duck homologue of mammalian IL-17D was first cloned from duck large intestine cDNA. The cloned duIL-17D sequence contained a 624-bp open reading frame (ORF) predicted to encode a putative 207 amino acid protein with a calculated molecular weight of $23 \mathrm{kDa}$ (non-glycosylated) and an isoelectric point of 8.97. The duIL-17D amino acid sequence contained a signal sequence (amino acids $1-22$ ), one potential $\mathrm{N}$ linked glycosylation site (Asn-X-Ser/Thr) in the extracellular region, and eight cysteine residues involved in the interchain disulfide bond formation that is conserved in the IL-17 family (Starnes et al., 2002; Moseley et al., 2003) (Fig. 1A and B). Interestingly, the duIL17D protein identified in this study is 91 amino acids longer than the previously cloned chicken IL-17D (GenBank accession number ABU51881) (Hong et al., 2008), which is predicted to encode a putative 116 amino acid protein with a calculated molecular weight of $13.3 \mathrm{kDa}$, suggesting the possibility that the ABU51881 clone may be a short isoform or a truncated form of IL-17D.

To confirm this possibility, chicken IL-17D (chIL-17D) was screened from chicken skeletal muscle cDNA based on the duIL-17D cDNA sequence, and a full-length chIL-17D cDNA was also identified in this study (GenBank accession number ALM23739). The chIL-17D sequence that was similar to duIL-17D contained a 621-bp ORF predicted to encode a putative 206 amino acid protein with a calculated molecular weight of $23 \mathrm{kDa}$ (non-glycosylated) and an isoelectric point of 8.97. The chIL-17D amino acid sequence contained a signal sequence (amino acids $1-22$ ), one potential $N$ linked glycosylation site in the extracellular region, and eight cysteine residues (Supplementary Fig. S1A). Multiple amino acid alignment revealed that, compared to the chIL-17D cDNA cloned in this study, the previously reported cDNA (GenBank accession no. ABU51881; Hong et al., 2008) was completely identical only to amino acids 102-206 of full-length chIL-17D (Supplementary 
Fig. S1B). Amino acid sequence comparison using Clustal Omega indicated that duIL-17D shares approximately $94.7 \%$ identity with its chicken counterpart identified in this work. The duIL-17D shares 62.6-68.4\% identity with mammalian homologues and $52.1-53.1 \%$ identity with its piscine counterparts (Fig. 1C).

\subsection{Distribution of duIL-17D mRNA in healthy tissues and mitogen- activated splenic lymphocytes}

We performed qRT-PCR analysis to examine the expression of duIL-17D transcript in various healthy tissues and in mitogenstimulated splenic lymphocytes (Fig. 1D and E). The expression of duIL-17D transcripts was detected in all tissues, especially the heart, which exhibited a relatively high expression level. Healthy lymphoid tissues such as bursa, thymus, and spleen generally expressed very low levels of duIL-17D transcript (Fig. 1D). This result was somewhat consistent with that of chIL-17D, which showed very low expression levels in chicken lymphoid tissues such as bursa, thymus, cecal tonsil, and spleen (Supplementary Fig. S1C). As shown in Fig. 1E, duIL-17D mRNA transcript was generally upregulated at all time points of ConA, LPS and poly I:C stimulation examined in splenic lymphocytes compared with unstimulated and cultured lymphocytes.

\subsection{Molecular weight and bioactivity of recombinant duIL-17D}

A construct expressing FLAG-tagged duIL-17D or empty vector was transiently transfected into COS-7 cells. Molecular weights of duIL-17D were identified in PNGase F-treated COS-7 cells transfected with a FLAG-tagged duIL-17D construct. A $23 \mathrm{kDa}$ protein (lower arrow; calculated molecular weight of $23 \mathrm{kDa}$ ) is likely the backbone of dulL-17D, and the $27 \mathrm{kDa}$ protein (upper arrow) represents an $\mathrm{N}$-linked glycosylated form of the protein. Induction of proinflammatory cytokines is a characteristic of IL-17 family members (Iwakura et al., 2011; Pappu et al., 2011). Thus, the supernatant was used for investigating the biological activity of recombinant duIL-17D in primary DEF. When we compared the effect of adding tissue culture supernatant from COS-7 cells that were transiently transfected with FLAG-tagged duIL-17D to supernatant from cells transfected with the empty vector, we found that supernatant containing duIL-17D induced significantly higher expression of IL- $1 \beta$, IL-6, and IL- 8 transcripts, but not TGF- $\beta$ (Fig. 1G).

\subsection{Downregulation of duIL-17D in R. anatipestifer-infected ducks}

Although many studies have demonstrated the biological function of IL-17 family cytokines such as IL-17A in different animal and disease models, only a few have investigated the role of IL-17D in host defense against major pathogens. $R$. anatipestifer, one of most harmful bacterial pathogens of ducks, has been implicated in a range of diseases, including fibrinous pericarditis, polyserositis, and septicemia (Ruiz and Sandhu, 2013). In an initial effort to determine the expression pattern of duIL-17D mRNA, ducks were infected intramuscularly with $R$. anatipestifer. Compared to uninfected ducks, the level of duIL-17D mRNA remained unchanged or was significantly downregulated in the liver and spleen (Fig. 2A). However, dulL-17A mRNA was upregulated in both liver and spleen compared to that of healthy controls (Fig. 2B). On days 7 and 10, IL$17 \mathrm{~A}$ expression was dramatically reduced in the spleen of $R$. anatipestifer-infected ducks (Fig. 2B). To confirm infection, bacterial burden was monitored in the spleen and liver post-infection, and was found in both organs of infected ducks (Fig. 2C).

\section{Discussion}

IL-17D is a proinflammatory cytokine of the IL-17 cytokine family. To date, IL-17D is the least studied member of this family. Here, a full-length cDNA encoding the duck homologue of mammalian IL-17D was cloned and characterized. We also found that duIL-17D exhibits higher conservation than duIL-17A and duIL$17 \mathrm{~F}$ between mammals and birds. The putative protein of duIL-17D showed $62.6-68.4 \%$ and $94.7 \%$ identities with the corresponding mammalian homologues and chIL-17D identified in this study, respectively. Our previous studies indicated that dulL-17A and duIL-17F shared $46-47 \%$ and $37-46 \%$ identities with mammalian homologues, respectively. Furthermore, duIL-17A and duIL-17F exhibited $84 \%$ and $77.5 \%$ identities with chIL-17A and chIL-17F, respectively (Min and Lillehoj, 2002; Yoo et al., 2009; Kim et al., 2012, 2015). Multiple sequence alignment revealed the presence of eight cysteine residues potentially involved in the formation of disulfide bonds are conserved in both avian and mammalian IL17D. Eight of the conserved cysteine residues were also found in IL-17D of Atlantic salmon (Kumari et al., 2009). However, duIL-17A and dulL-17F possess six of the conserved cysteine residues (Yoo et al., 2009; Kim et al., 2015), indicating that the avian IL-17D sequences contained two additional cysteines compared to the duIL$17 \mathrm{~A}$ and IL-17F sequences. Thus, our data suggest that the newly cloned IL-17D cDNA is an avian ortholog of mammalian IL-17D.

The IL-17 family cytokines induce the production of proinflammatory cytokines, chemokines, and matrix metalloproteinases (Weaver et al., 2007; Iwakura et al., 2011). Similar to the other IL-17 family members that we previously cloned, namely duIL-17A and duIL-17F (Yoo et al., 2009; Kim et al., 2015), duIL-17D treatment led to increased expression of IL-1 $\beta$, IL-6, and IL- 8 in primary DEF. However, duIL-17D did not induce the expression of duck TGF- $\beta$. IL17D induced IL-6, IL-8, and GM-CSF production in human endothelial cells (Starnes et al., 2002), as well as IL-1 $\beta$, CXCL-8, and TNF$\alpha$ mRNA expression in grass carp primary head kidney cells (Du et al., 2014).

Like in human and fish species (Starnes et al., 2002; Kumari et al., 2009; Korenaga et al., 2010; Du et al., 2014), constitutive expression of duIL-17D (Fig. 1D) and chIL-17D (Supplementary Fig. S1C) transcripts was ubiquitous in both bird organs examined. Duck and chicken IL-17D transcripts were expressed at a relatively low level in healthy lymphoid tissues such as spleen, thymus, and bursa. These observations are in accordance with the low abundance of IL-17D mRNA in the spleen of grass carp (Du et al., 2014), Atlantic salmon (Kumari et al., 2009), broiler chickens (Hong et al., 2008), and humans (Starnes et al., 2002). However, IL-17D was expressed at different levels across these species in the thymus; IL-17D was expressed at low levels in grass carp and human while Atlantic salmon and broiler chickens showed moderate or high expression levels. Relatively high expression of duIL-17D (Fig. 1D) and chIL-17D (Supplementary Fig. S1C) transcripts has been noted in the heart, which is consistent with findings in humans (Starnes et al., 2002) and teleost fish tongue sole (Chi and Sun, 2015). To investigate whether the mRNA expression profiles of duIL-17D could be modulated, splenic lymphocytes were stimulated in vitro and duIL-17D mRNA was assessed (Fig. 1E). The level of duIL-17D expression remained unchanged or increased significantly in splenic lymphocytes stimulated with ConA, LPS, or poly I:C. Similarly, reports have demonstrated that, after LPS stimulation, IL-17D expression was unchanged or increased significantly in head kidney cells of pufferfish (Korenaga et al., 2010) and significantly induced at all time points examined in head kidney leukocytes of grass carp (Du et al., 2014). These findings suggest that in vitro expression profiles of IL-17D may be similar between ducks and fish. 


\section{A}

ATGCAGCGAGGCAGGGTGCTGGCGGCGCTGCTGTGCGCGGCGCTGCTGCCGCTGCCCTCGGACGCCGCCAAAGCTCCCAAGCGGCCGGCTCGGCCCCGGGGCTGCGGGGAGAGGCCCGAG 120

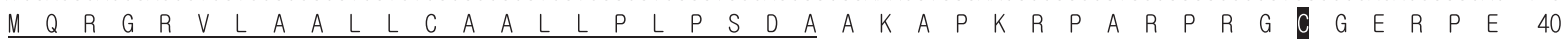
GAGCTGCTGGAGCAGCTGTACGGGCGGCTGGCCGCCGGCATGCTCAGCGCCTTCCACCACACGCTGCAGCCCGAGCCGCCCGGCCGCCAGCAGCACAACGCCAGCTGCCCCGCGGGGGCA 240

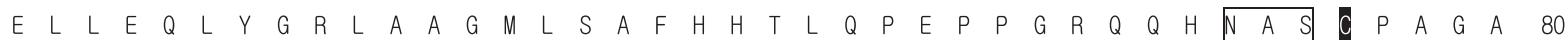
CGGCCGGCCGGCGACAAGAGGCTGCGGCTGCCCGTCAACATGCGCAGCGCCTCGCCCTGGGCGTACAGAATTTCATACGATCCCACGAGATACCCTAAGTACATTCCCGAAGCGTACTGC 360

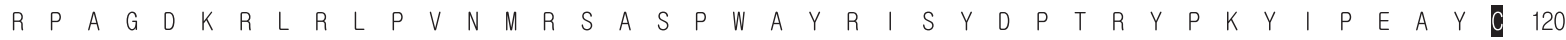
CTGTGCAAGGGCTGCCTCATGGGGATCTTCGGCGAGGAGAATTTCCACTTCCGCAGCACCCCCGTGTACATGCCCACCGTCATCCTCCGCCGCACCTCGTCCTGTGCCGGGGGCCGCTAC 480

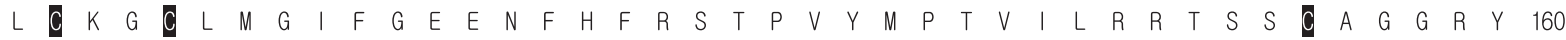
GTGTACACGGAAGACTACGTCACTATCCCCGTGGGCTGCACTTGTGTCCCCGAGCCAGAGAAAGAGGCCGAAAGCGTAAATTCCAGCATAGATAAGCAAGAAATGAAGTTGCTCGTAAAC 600

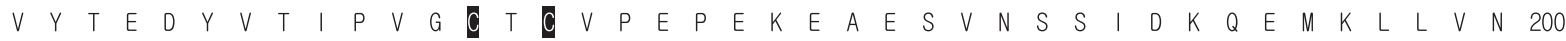
CAGAACAAGCCATCTTCAGAATGA

Q $N$ K $K$ P S S S E -

B

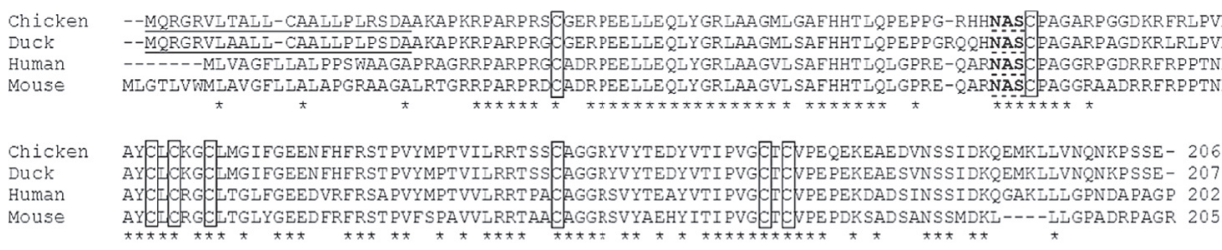

$\mathrm{C}$

\begin{tabular}{|c|c|c|c|c|c|c|c|c|c|c|c|}
\hline \multirow[b]{2}{*}{ duIL-17D } & \multicolumn{6}{|c|}{ human/mouse } & \multicolumn{3}{|c|}{ chicken/duck } & \multirow{2}{*}{$\begin{array}{c}\text { salmon/trout } \\
\text { IL-17D }\end{array}$} & \multirow{2}{*}{$\begin{array}{c}\text { frog } \\
\text { IL-17D }\end{array}$} \\
\hline & IL-17A & IL-17B & IL-17C & IL-17D & IL-17E & IL-17F & IL-17A & IL-17F & IL-17D & & \\
\hline & $21.2-21.4$ & $21.6-25.8$ & $23.2-26$ & $62.6-68.4$ & $16.5-22.4$ & $17.5-18.1$ & $22.0-25.2$ & $20.6-22.6$ & 94.7 & $52.1-53.1$ & 61.7 \\
\hline
\end{tabular}

D

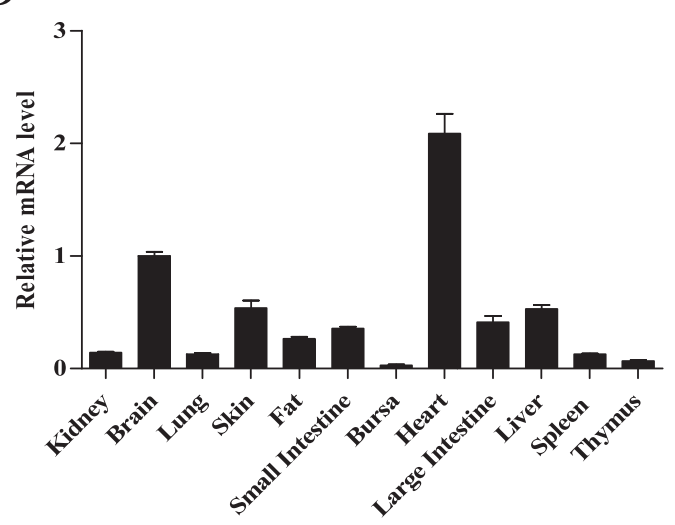

E

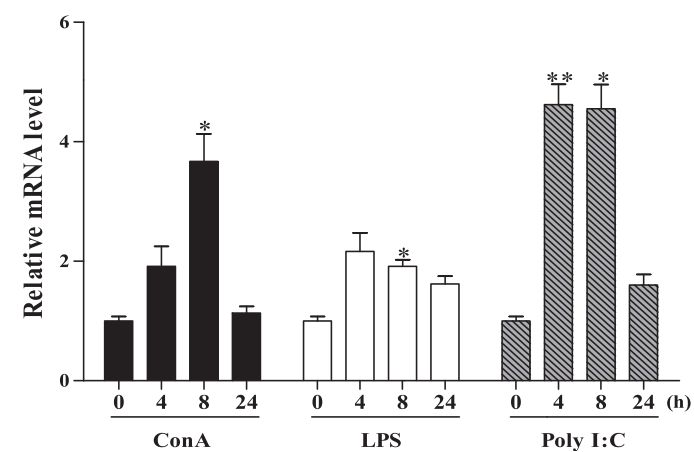

F

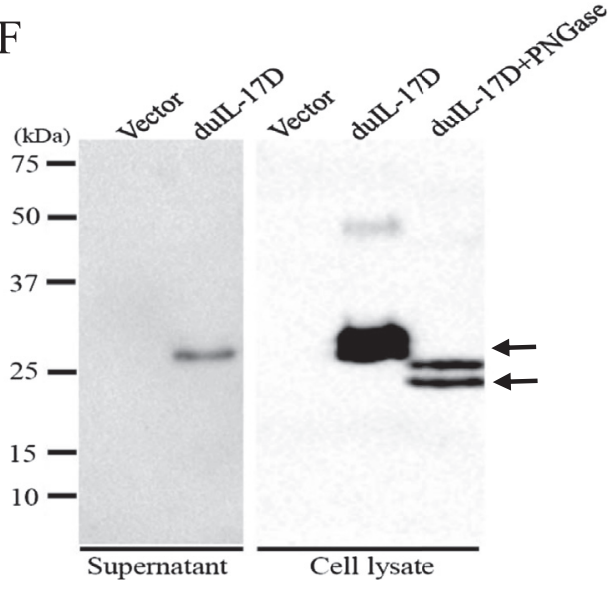

G

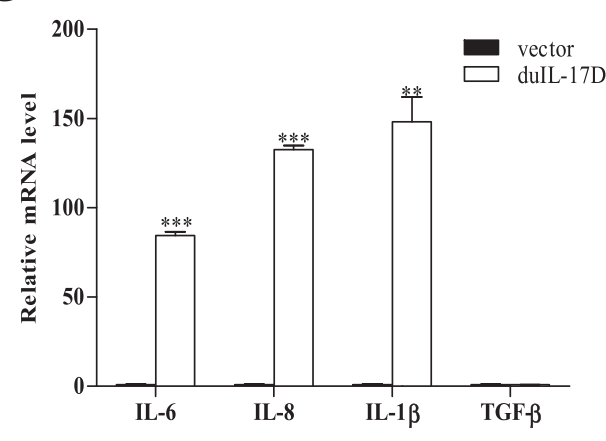

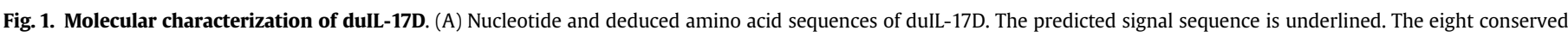

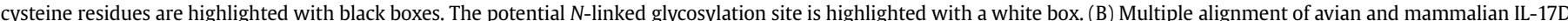

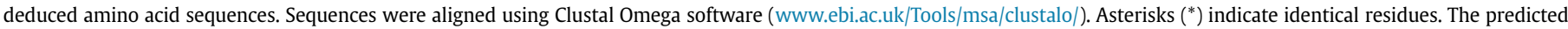

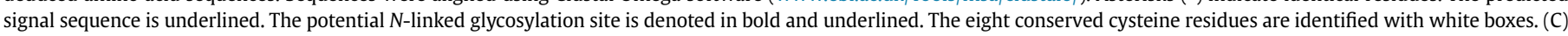

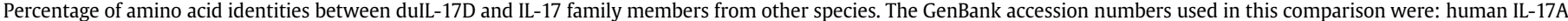

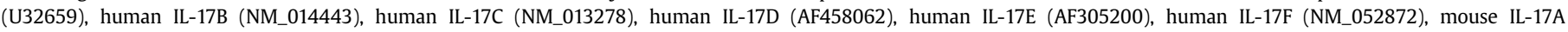

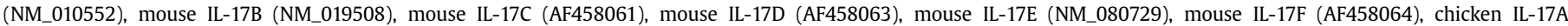

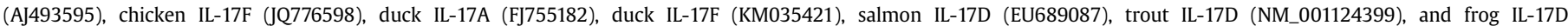

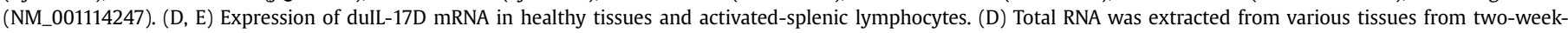


A

Spleen

Liver
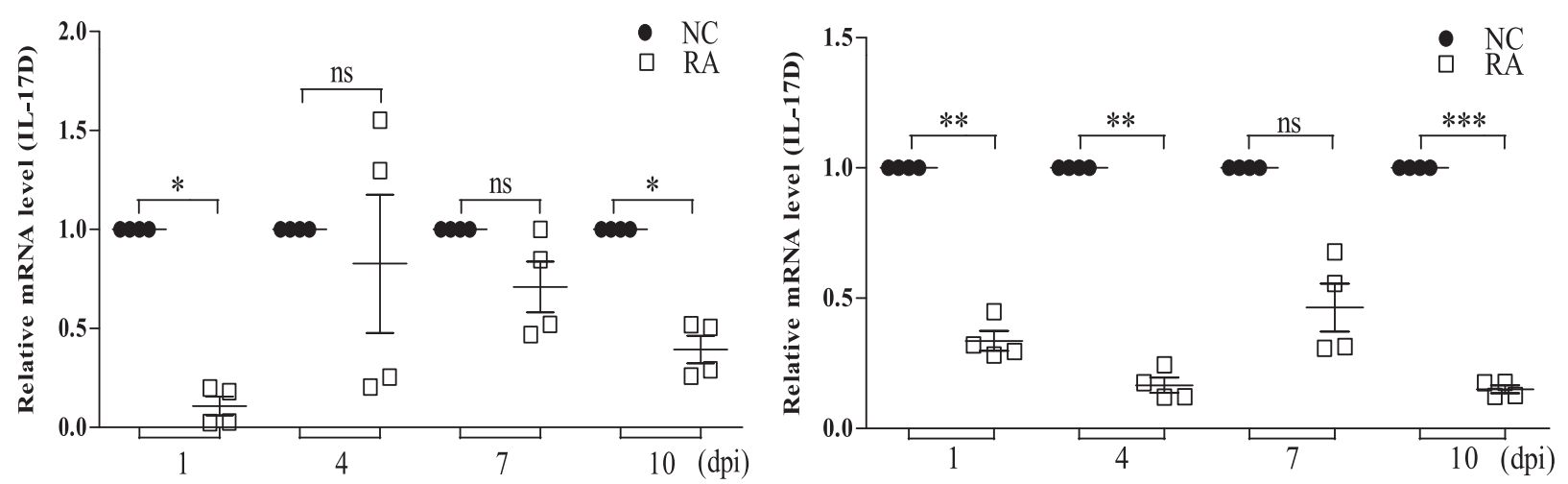

$\mathrm{B}$
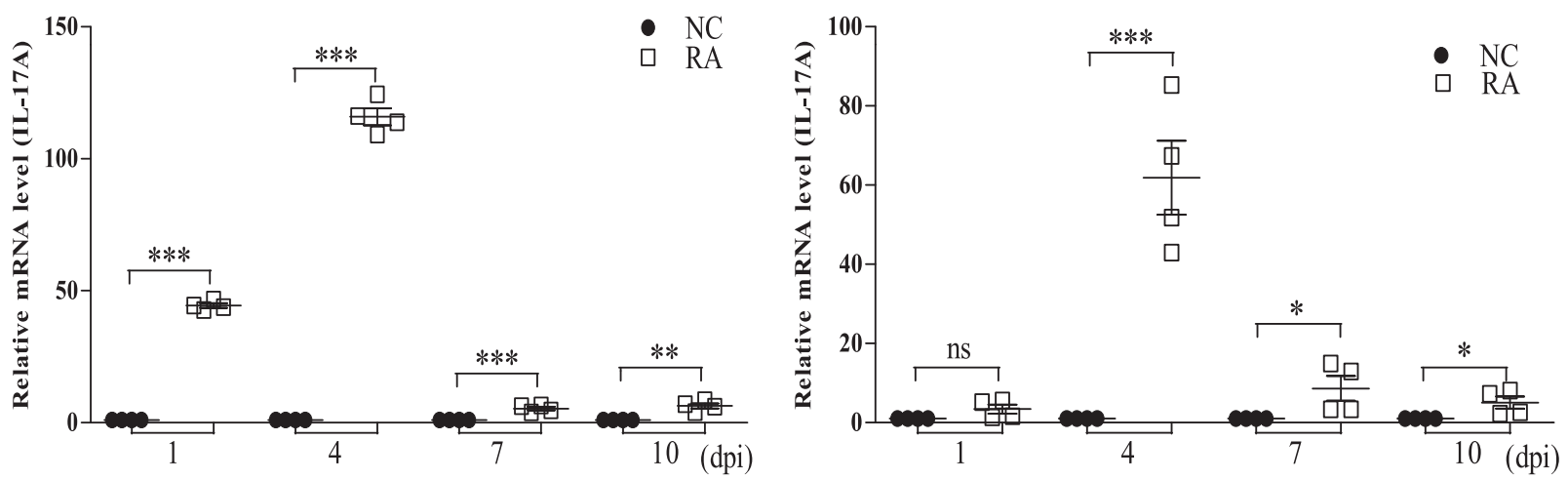

$\mathrm{C}$
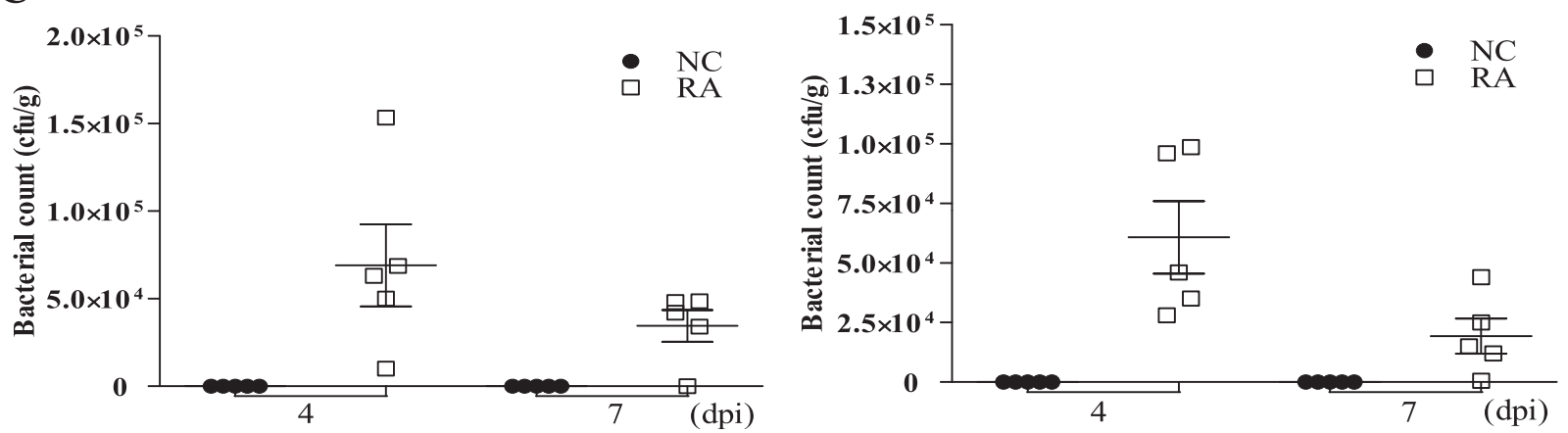

Fig. 2. Expression profiles of duIL-17D and duIL-17A mRNA in R. anatipestifer-infected ducks. Fourteen-day-old ducks were infected via intramuscular injection with $5 \times 10^{7}$ colony-forming units (cfu) of R. anatipestifer. The liver and spleen of $R$. anatipestifer-infected ducks were collected on days $1,4,7$, and 10 post-infection. Expression levels of duIL-17D (A) and duIL-17A (B) transcript were quantified by qRT-PCR. Gene expression levels were normalized with $\beta$-actin and calibrated with expression found in the uninfected group. Error bars represent the mean $\pm \mathrm{SE}(\mathrm{n}=4) .{ }^{*} P<0.05,{ }^{* *} P<0.01$, or ${ }^{* * *} P<0.001$ was considered significant compared to the uninfected group, which was used as a negative control (NC). (C) Bacterial load of R. anatipestifer in the liver and spleen. Five ducks were sacrificed at each time point and then the liver and spleen were aseptically collected for bacterial recovery. One representative experiment of two independent experiments performed with similar results is shown. RA, R. anatipestifer; NC, negative control; dpi, days post-infection; ns, no statistical significance.

old healthy ducks. Tissue samples were pooled from five ducks and were subjected to qRT-PCR analysis. Gene expression levels were normalized with $\beta$-actin and calibrated with brain tissue expression. Data represent the mean \pm SE from two independent experiments. (E) Splenic lymphocytes were isolated from two-week-old ducks, and activated with $10 \mu \mathrm{g} / \mathrm{ml}$ ConA, $10 \mu \mathrm{g} / \mathrm{ml}$ LPS, or $25 \mu \mathrm{g} / \mathrm{ml}$ poly I:C for the indicated times. Gene expression levels were normalized to $\beta$-actin and calibrated with unstimulated and cultured splenic lymphocytes. The y-axis represents the fold change in expression of duIL-17D in activated lymphocytes compared to unstimulated and cultured splenic lymphocytes. All data represent the mean \pm SE from two independent experiments. ${ }^{*} P<0.05$ or ${ }^{* *} P<0.01$ was considered to be statistically significant. (F, G) Molecular weight and bioactivity of duIL17D. (F) Detection of duIL-17D protein by western blot analysis. COS-7 cells were transiently transfected with duIL-17D-FLAG or vector pcDNA 3.1. Supernatants and cell lysates were collected after $48 \mathrm{~h}$ and separated by SDS-PAGE under reducing conditions. Cell lysates were deglycosylated by $100 \mathrm{U}$ peptide- $\mathrm{N}$-glycosidase F (PNGase-F) at $37{ }^{\circ} \mathrm{C}$ for $2 \mathrm{~h}$. The arrows indicate specific bands. One representative experiment of two independent experiments performed with similar results is shown. (G) Induction of proinflammatory cytokines in primary DEF. Primary DEF was obtained from ten-day-old embryos as previously described (Kim et al., 2015) and then stimulated with the conditioned medium from COS-7 cells transfected with dulL-17D or empty vector (pcDNA 3.1+) for $6 \mathrm{~h}$ at $41{ }^{\circ} \mathrm{C}$ in $5 \% \mathrm{CO}_{2}$. Total RNA was extracted and then cytokine gene expression was analyzed by qRTPCR. Expression levels were normalized to those of $\beta$-actin. Data represent the mean \pm SE from two independent experiments. ${ }^{* *} P<0.01$ or ${ }^{* * *} P<0.001$ was considered to be statistically significant. 
$R$. anatipestifer is a Gram-negative, non-motile, non-sporeforming, rod-shaped, and extracellular bacterium that causes one of the most economically harmful and contagious diseases in duck farms worldwide (Hu et al., 2012; Zhou et al., 2013). R. anatipestifer infection causes polyserositis, septicemia, and meningitis, and is associated with a $5 \%-75 \%$ mortality rate depending on the virulence of the strain (Pathanasophon et al., 2002). This pathogen enters through skin wounds or the respiratory tract and is carried to various organs, including the spleen and liver (Ruiz and Sandhu, 2013). In this study, duIL-17D, unlike duIL-17A, was largely downregulated in the liver and spleen during $R$. anatipestifer infection compared to uninfected ducks. Previous studies with pathogenic bacteria and parasites showed a variation in IL-17D expression pattern (unresponsive, decrease or increase) depending on the tissues examined after infection (Hong et al., 2008; Kumari et al., 2009; Du et al., 2014). In grass carp, infection with Aeromonas hydrophila for 1 and 3 days led to increased IL-17D expression in the head kidney; expression of this transcript returned to basal levels at day 7 (Du et al., 2014). IL-17D expression in Atlantic salmon was significantly increased in the head kidney after Aeromonas salmonicida infection and returned to basal levels at day 4. In contrast, IL-17D was unchanged in the spleen during the infection (Kumari et al., 2009). The expression level of IL-17D was unchanged in the spleen of teleost fish tongue sole during Vibrio harveyi infection (Chi and Sun, 2015). In broiler chickens infected with the intracellular parasite E. maxima, the level of IL-17D mRNA was increased in the jejunum, bursa, lung, and spleen, but decreased in thymus (Hong et al., 2008). Taken together, these observations suggest the possibility that expression levels of IL-17D in the spleen of ducks and fish may be unchanged or downregulated to facilitate effective host immunity during pathogenic bacterial infections. It is interesting to note that IL-17D treatment showed a suppressive effect on myeloid progenitor cell proliferation (Starnes et al., 2002). However, discrepancies in IL-17D expression levels occur in the spleen of Eimeriainfected chickens where its expression was increased. This discrepancy is likely attributable to assessing different pathogens with different pathogenic mechanisms. We employed the bacterial pathogen $R$. anatipestifer, whereas Hong et al. (2008) used an intracellular parasite E. maxima. While R. anatipestifer is translocated to various organs, including the spleen and liver (Ruiz and Sandhu, 2013), E. maxima targets the enterocytes and causes significant damage to the intestinal epithelial layer (Min et al., 2013; Chapman, 2014). Further investigation is needed to assess the differential expression of duIL-17D in response to various pathogens and its differential functions in host immunity and defense mechanisms against those pathogens.

In summary, we cloned duIL-17D and examined its expression patterns and functions. Molecular analysis indicated that duIL-17D possesses several conserved features characteristic of the Th17 family of cytokines. When compared to duIL-17A and duIL-17F, duIL-17D possesses distinct characteristics in tissue distribution and expression patterns in mitogen-activated splenic lymphocytes and in $R$. anatipestifer-infected ducks, indicating that duIL-17D is a unique member of the IL-17 family of cytokines.

\section{Acknowledgements}

This research was supported by Basic Science Research Program through the National Research Foundation of Korea (NRF) funded by the Ministry of Education (2015R1D1A1A02059953).

\section{Appendix A. Supplementary data}

Supplementary data related to this article can be found at http:// dx.doi.org/10.1016/j.dci.2016.04.002.

\section{References}

Chapman, H.D., 2014. Milestones in avian coccidiosis research: a review. Poult. Sci. 93, 501-511.

Chi, H., Sun, L., 2015. Comparative study of four interleukin 17 cytokines of tongue sole Cynoglossus semilaevis: genomic structure, expression pattern, and promoter activity. Fish Shellfish Immunol. 47, 321-330.

Du, L., Qin, L., Wang, X., Zhang, A., Wei, H., Zhou, H., 2014. Characterization of grass carp (Ctenopharyngodon idella) IL-17D: molecular cloning, functional implication and signal transduction. Dev. Comp. Immunol. 42, 220-228.

Gibson, M.S., Fife, M., Bird, S., Salmon, N., Kaiser, P., 2012. Identification, cloning, and functional characterization of the IL-1 receptor antagonist in the chicken revea important differences between the chicken and mammals. J. Immunol. 189, 539-550.

Gu, C., Wu, L., Li, X., 2013. IL-17 family: cytokines, receptors and signaling. Cytokine $64,477-485$.

Hong, Y.H., Lillehoj, H.S., Park, D.W., Lee, S.H., Han, J.Y., Shin, J.H., Park, M.S., Kim, J.K., 2008. Cloning and functional characterization of chicken interleukin-17D. Vet. Immunol. Immunopathol. 126, 1-8.

Hu, Q., Ding, C., Tu, J., Wang, X., Han, X., Duan, Y., Yu, S., 2012. Immunoproteomics analysis of whole cell bacterial proteins of Riemerella anatipestifer. Vet. Microbiol. 157, 428-438.

Hwang, S.Y., Kim, H.Y., 2005. Expression of IL-17 homologs and their receptors in the synovial cells of rheumatoid arthritis patients. Mol. Cells 19, 180-184.

Iwakura, Y., Ishigame, H., Saijo, S., Nakae, S., 2011. Functional specialization of interleukin-17 family members. Immunity 34, 149-162.

Jeong, J., Kim, W.H., Yeo, J., Fernandez, C.P., Kim, S., Lee, Y.J., Lillehoj, H.S., Min, W. 2014. Identification of alternatively spliced isoforms of interleukin-2/15 receptor $\beta$ chain in ducks. Vet. Immunol. Immunopathol. 162, 154-161.

Jeong, J., Kim, W.H., Fernandez, C.P., Kim, S., Kim, Y.H., Jang, H.K., Lillehoj, H.S. Woo, H.J., Min, W., 2015. Different strategies for producing naturally soluble form of common cytokine receptor $\gamma$ chain. Dev. Comp. Immunol. 48, $13-21$.

Johansen, C., Usher, P.A., Kjellerup, R.B., Lundsgaard, D., Iversen, L., Kragballe, K., 2009. Characterization of the interleukin-17 isoforms and receptors in lesional psoriatic skin. Br. J. Dermatol. 160, 319-324.

Kim, W.H., Jeong, J., Park, A.R., Yim, D., Kim, Y.H., Kim, K.D., Chang, H.H. Lillehoj, H.S., Lee, B.H., Min, W., 2012. Chicken IL-17F: identification and comparative expression analysis in Eimeria-infected chickens. Dev. Comp. Immunol. 38, 401-409.

Kim, W.H., Jeong, J., Park, A.R., Yim, D., Kim, S., Chang, H.H., Yang, S.H., Kim, D.H. Lillehoj, H.S., Min, W., 2014. Downregulation of chicken interleukin-17 receptor A during Eimeria infection. Infect. Immun. 82, 3845-3854.

Kim, W.H., Fernandez, C.P., Diaz, J.A., Jeong, J., Kim, S., Lillehoj, H.S., Chang, H.H., Min, W., 2015. Molecular cloning, characterization and mRNA expression of duck interleukin-17F. Vet. Immunol. Immunopathol. 164, 194-200.

Korenaga, H., Kono, T., Sakai, M., 2010. Isolation of seven IL-17 family genes from the Japanese pufferfish Takifugu rubripes. Fish Shellfish Immunol. 28, 809-818.

Kumari, J., Larsen, A.N., Bogwald, J., Dalmo, R.A., 2009. Interleukin-17D in Atlantic salmon (Salmo salar): molecular characterization, 3D modelling and promoter analysis. Fish Shellfish Immunol. 27, 647-659.

Liu, H., Wang, J., Si, J., Jia, J., Li, L., Han, C., Huang, K., He, H., Xu, F., 2012. Molecular cloning and in silico analysis of the duck (Anas platyrhynchos) MEF2A gene cDNA and its expression profile in muscle tissues during fetal development. Genet. Mol. Biol. 35, 182-190.

Mensikova, M., Stepanova, H., Faldyna, M., 2013. Interleukin-17 in veterinary animal species and its role in various diseases: a review. Cytokine 64, 11-17.

Min, W., Lillehoj, H.S., 2002. Isolation and characterization of chicken interleukin-17 cDNA. J. Interf. Cytokine Res. 22, 1123-1128.

Min, W., Kim, W.H., Lillehoj, E.P., Lillehoj, H.S., 2013. Recent progress in host immunity to avian coccidiosis: IL-17 family cytokines as sentinels of the intestinal mucosa. Dev. Comp. Immunol. 41, 418-428.

Moseley, T.A., Haudenschild, D.R., Rose, L., Reddi, A.H., 2003. Interleukin-17 family and IL-17 receptors. Cytokine Growth Factor Rev. 14, 155-174.

Onishi, R.M., Gaffen, S.L., 2010. Interleukin-17 and its target genes: mechanisms of interleukin-17 function in disease. Immunology 129, 311-321.

O'Sullivan, T., Saddawi-Konefka, R., Gross, E., Tran, M., Mayfield, S.P., Ikeda, H. Bui, J.D., 2014. Interleukin-17D mediates tumor rejection through recruitment of natural killer cells. Cell Rep. 7, 989-998.

Pappu, R., Ramirez-Carrozzi, V., Sambandam, A., 2011. The interleukin-17 cytokine family: critical players in host defence and inflammatory diseases. Immunology 134, 8-16.

Pappu, R., Rutz, S., Ouyang, W., 2012. Regulation of epithelial immunity by IL-17 family cytokines. Trends Immunol. 33, 343-349.

Pathanasophon, P., Phuektes, P., Tanticharoenyos, T., Narongsak, W., Sawada, T. 2002. A potential new serotype of Riemerella anatipestifer isolated from ducks in Thailand. Avian Pathol. 31, 267-270.

Ramirez-Carozzi, V., Sambandam, A., Luis, E., Lin, Z., Jeet, S., Lesch, J., Hackney, J., Kim, J., Zhou, M., Lai, J., Modrusan, Z., Sai, T., Lee, W., Xu, M., Caplazi, P., Diehl, L. de Voss, J., Balazs, M., Gonzalez Jr., L., Singh, H., Ouyang, W., Pappu, R., 2011. IL17C regulates the innate immune function of epithelial cells in an autocrine manner. Nat. Immunol. 12, 1159-1166.

Ruiz, J.A., Sandhu, T.S., 2013. Riemerella anatipestifer infection. In: Swayne, D.E., 
Glisson, J.R., McDougald, L.R., Nolan, L.K., Suarez, D.L., Nair, V. (Eds.), Diseases of Poultry, thirteenth ed. Wiley-Blackwell publication, USA, pp. 823-828.

Rychlik, I., Elsheimer-Matulova, M., Kyrova, K., 2014. Gene expression in the chicken caecum in response to infections with non-typhoid Salmonella. Vet. Res. 45, 119.

Shanmugasundaram, R., Selvaraj, R.K., 2012. Regulatory T cell properties of thymic CD4+CD25+ cells in ducks. Vet. Immunol. Immunopathol. 149, 20-27.

Song, X., Qian, Y., 2013. IL-17 family cytokines mediated signaling in the pathogenesis of inflammatory diseases. Cell. Signal 25, 2335-2347.

Starnes, T., Broxmeyer, H.E., Robertson, M.J., Hromas, R., 2002. Cutting edge: IL-17D, a novel member of the IL-17 family, stimulates cytokine production and inhibits hemopoiesis. J. Immunol. 169, 642-646.

Stewart, C.R., Keyburn, A.L., Deffrasnes, C., Tompkins, S.M., 2013. Potential directions for chicken immunology research. Dev. Comp. Immunol. 41, 463-468.

Weaver, C.T., Hatton, R.D., Mangan, P.R., Harrington, L.E., 2007. IL-17 family cytokines and the expanding diversity of effector $\mathrm{T}$ cell lineages. Annu. Rev. Immunol. 25, 821-852.
Wei, L., Cui, J., Song, Y., Zhang, S., Han, F., Yuan, R., Gong, L., Jiao, P., Liao, M., 2014a. Duck MDA5 functions in innate immunity against H5N1 highly pathogenic avian influenza virus infections. Vet. Res. 45, 66.

Wei, S., Liu, X., Gao, M., Zhang, W., Zhu, Y., Ma, B., Wang, J., 2014b. Cloning and characterization of goose interleukin-17A cDNA. Res. Vet. Sci. 96, 118-123.

Yoo, J., Jang, S.I., Kim, S., Cho, J.H., Lee, H.J., Rhee, M.H., Lillehoj, H.S., Min, W., 2009. Molecular characterization of duck interleukin-17. Vet. Immunol. Immunopathol. 132, 318-322.

Zhao, G.H., Cheng, W.Y., Wang, W. Jia, Y.O., Fang, Y.Q., Du, S.Z., Yu, S.K., 2014. The expression dynamics of IL-17 and Th17 response relative cytokines in the trachea and spleen of chickens after infection with Cryptosporidium baileyi. Parasit. Vectors 7, 212.

Zhou, Z., Li, X., Xiao, Y., Wang, X., Tian, W., Peng, X., Bi, D., Sun, M., Li, Z., 2013. Gene expression responses to Riemerella anatipestifer infection in the liver of ducks. Avian Pathol. 42, 129-136. 